[Cierre de edición 12 de noviembre del 2010]

\title{
Atención a la diversidad desde una visión interdisciplinaria: resultados del Trabajo Final de Graduación de un grupo de estudiantes de las carreras de Educación Preescolar y Especial
}

\section{Attention to Diversity from an Interdisciplinary Perspective: Results from the Final Graduation Project from a Group of Students Majoring in Preschool Education and Special Education}

\author{
Paola Delgado Obregón ${ }^{1}$ \\ Estudiante de la Universidad Nacional \\ Heredia, Costa Rica \\ Grettel Hernández Barboza $a^{2}$ \\ Jardín de Niños San Alfonso \\ Alajuela, Costa Rica \\ Heidy León Arce \\ División de Educación Básica del Centro de Investigación y Docencia en Educación \\ Universidad Nacional \\ Heredia, Costa Rica \\ Laura Morera Calvo ${ }^{4}$ \\ Estudiante de la Universidad Nacional \\ Heredia, Costa Rica \\ Silvia Elena Morera Villarebia ${ }^{5}$ \\ Jardín de Niños Ludoteca y Guardería Arca de Noé \\ Alajuela, Costa Rica \\ Roxana Obregón Vargas 6 \\ Escuela Llorente de Flores, \\ Heredia, Costa Rica
}

Recibido 23 de marzo de $2010 \bullet$ Aceptado 24 de junio de 2010

\footnotetext{
Licenciada en Pedagogía con énfasis en educación Preescolar. Bachiller en Educación Preescolar. Cursó el programa de Inglés como Segunda Lengua en la Universidad de Wisconsin Eau Claire, Estados Unidos. Tiene experiencia docente en instituciones costarricenses, en las que ha trabajado en educación preescolar y enseñanza del inglés. Correo electrónico: poadelgado@gmail.com
}

2 Licenciada en Educación Preescolar. Tiene experiencia docente en instituciones privadas costarricenses. Labora en el Jardín de Niños San Alfonso, como docente del nivel de kinder. Correo electrónico: gre.mar03@gmail.com.

3 Licenciada en Educación Especial con énfasis en Integración. Bachiller en Educación Especial. Diplomado en Ciencias de la Educación con concentración en Educación Básica I y II ciclos de la Universidad Nacional. Tiene experiencia docente en instituciones costarricenses. Labora en la División de Educación Básica del Centro de Investigación y Docencia en Educación, como académica de la carrera de Educación Especial y como miembro del proyecto Mejoramiento en el Marco de la Acreditación. Labora para el Ministerio de Educación Pública en el área de Retardo Mental en el servicio de Estimulación Temprana. Cursa la Maestría en Pedagogía con Énfasis en Diversidad de los Procesos Educativos. Correo electrónico: heidyleon15@hotmail.com

4 Licenciada en Pedagogía con énfasis en Educación Preescolar. Bachiller en Pedagogía con énfasis en Educación Preescolar. Diplomado en Pedagogía con énfasis en Educación Preescolar de la Universidad Nacional, Costa Rica. Tiene experiencia docente en instituciones privadas costarricenses. Correo electrónico: laumorera02@gmail.com

5 Licenciada en Pedagogía con énfasis en Educación Preescolar. Bachiller en Pedagogía con énfasis en Educación Preescolar. Diplomado en Pedagogía con énfasis en Educación Preescolar, Universidad Nacional. Tiene experiencia docente en instituciones privadas costarricenses, en las que ha trabajado como asistente de educación preescolar y docente encargada de educación preescolar. Actualmente labora en el Jardín de niños Ludoteca y Guardería Arca de Noé, trabajado con niños entre dos años y seis meses a tres años y seis meses. Correo electrónico: silviamorerav@yahoo.com.mx

6 Licenciada en Educación Especial con énfasis en Integración. Bachiller en Educación Especial. Diplomado en Ciencias de la Educación con concentración en Educación Básica I y II ciclos de la Universidad Nacional. Tiene experiencia docente por 14 años en instituciones públicas y privadas costarricenses, en las que ha trabajado en educación primaria y en servicios de Educación Especial .Actualmente labora para el Ministerio de Educación Pública en el área de Retardo Mental en el servicio de Aula Integrada en la escuela Llorente de Flores, Heredia, Costa Rica. Correo electrónico: roxanaov@gmail.com 
Resumen. En la actualidad, el tema sobre diversidad está siendo estudiado, particularmente, en el ámbito de la formación docente. Con el fin de entender este concepto y de conocer los retos que demanda este estudio, por medio de la investigación-acción se busca encontrar un panorama real de cómo la diversidad es atendida en los contextos rurales guanacastecos. Esto se logra identificando aquellas maneras que permitan orientar la labor docente, para lo cual se toma en consideración la planificación educativa y la participación de los diversos sectores involucrados en los procesos de enseñanza y de aprendizaje.

Palabras clave. Diversidad, interdisciplinariedad, gestión, negociación, enfoque multidimensional, Educación Preescolar, Educación Especial.

Abstract. Nowadays, the topic of diversity is being studied, particularly in the field of the formation of future educators, where it is clearly evident in each one of the students. In order to understand this concept and meet the challenges it demands, this investigation, through the experience of action research, looks for a real picture of how this diversity is served in Guanacaste's rural contexts. This is accomplished by identifying those ways to guide a better teachers' work, and by taking into account the educational planning and the participation of the different sectors involved in the process of teaching and learning.

Key words. Diversity, Interdisciplinary, Management, Negotiation, Multidimensional Approach, Preschool Education, Special Education.

\section{Hacia una experiencia nueva y enriquecedora}

Las universidades, sean públicas o privadas, constituyen uno de los pilares fundamentales en la formación integral de los futuros profesionales. Al analizar el papel tan necesario que cumplen para la sociedad, desde el inicio hasta la culminación de una carrera, resulta interesante visualizar cómo los Trabajos Finales de Graduación (TFG) deben velar por el desarrollo de aquellas experiencias integradoras en la formación profesional, y no que se conviertan en meros requisitos para la consecución de un título.

Con base en una investigación titulada Negociación, gestión e intervención de las estudiantes de las carreras de Educación Especial y Educación Preescolar de la Sede Central y la Sede Chorotega de la Universidad Nacional, en los procesos formativos de los docentes de las escuelas Río Ora, 26 de febrero de 1886, Lajas y el Jobo, del circuito 01, 05 y 07 de la provincia de Guanacaste que atiende niños con necesidades educativas especiales (Delgado, Hernández, Morera C., Morera V. y Obregón, 2009), en la cual participaron estudiantes de la carrera de Educación Preescolar y Educación Especial, se propuso abordar la temática de los aprendizajes a partir de los Trabajos Finales de Graduación.

La investigación tuvo como propósito facilitar el proceso de inclusión del grupo de docentes que atienden niños y niñas con necesidades educativas especiales (NEE), y promover en este las habilidades de gestión y de negociación para una asertiva intervención; asimismo, promover la participación de otros sectores (la comunidad, la familia y los mismos niños y niñas), que, de igual forma, influyen en la formación, el desarrollo y el aprendizaje del estudiantado.

Es necesario destacar que el estudio estaba orientado como un proyecto de extensión, el cual buscaba, precisamente, involucrar a estudiantes de TFG para que desarrollaran sus trabajos desde una perspectiva más participativa y lograr que impactaran en los contextos de estudio, situación a la que no están acostumbrados los estudiantes y las estudiantes.

Algunos de los TFG del grupo estudiantil se orientan al análisis de determinadas temáticas, las cuales suelen plantear propuestas que no siempre son llevadas a la práctica. Por ello, parece 
interesante abocarse al desarrollo de una investigación que involucre a los proponentes y las proponentes en un análisis temático desde la realidad de la comunidad y con una visión más participativa.

La investigación de la que se partió, en este caso, tiene similitudes con ese proceso; pero como se detallará más adelante, y se hará evidente, fue una experiencia que impactó, positivamente, en diversos sectores, inclusive a las mismas investigadoras, pues promovió un aprendizaje significativo enriquecedor para su vida profesional.

\section{Justificación}

La acepción de diversidad que las investigadoras asumen considera la diferencia como un valor positivo; por lo que toda diferencia individual debe ser considerada en los procesos educativos. Las necesidades educativas producto de estas diferencias se convierten en elementos fundamentales para la planificación educativa, sean estas derivadas de condiciones sociales, culturales, económicas o de discapacidad.

En Costa Rica, si bien es cierto se ha avanzado en torno al concepto que se tiene sobre discapacidad, en el subconsciente de muchos costarricenses aún pesan los miedos, la lástima y el rechazo hacia estas personas. En ese sentido, no es nuevo que alrededor de la atención de las personas con NEE, en el sistema educativo nacional, se encuentre vigente un imaginario que va desde la negación de atender las NEE por parte de cuerpo docente, hasta la exacerbación de la especialización de aquellos que son llamados, históricamente, a atender esta población.

De esta circunstancia, nace el interés de incentivar, mediante la investigación, el trabajo interdisciplinario, no sólo entre docentes en ejercicio, sino, también, en las autoras de este escrito, las cuales se han formado a partir de una concepción de Educación por disciplinas. Lo anterior, con el fin de promover mecanismos que sensibilicen a todos los profesionales en la atención a la diversidad.

En los ámbitos laborales, surge la preocupación por la independencia del quehacer de las distintas disciplinas educativas, la realidad en las normativas y los procedimientos de los profesionales en Educación Especial, Educación Preescolar y I y II Ciclos, pues en cada una de ellas se dificulta el proceso de trabajo en equipo y de coordinación con miras a un mismo objetivo, es decir, interdisciplinariedad, ya que en su formación se les ha dado poca oportunidad para vivenciar esta experiencia. Esta situación se suma a una debilidad en la capacidad de gestión y de negociación en los procesos educativos y, por ende, en una asertiva intervención.

Por tanto, es de suma importancia incorporar en el perfil del profesional docente algunas habilidades de negociación y de gestión facilitadoras de nuevas propuestas de trabajo, para desempeñar acciones que favorezcan una educación más accesible para todos. Además se requiere que el sistema educativo promueva cambios significativos a favor de la diversidad y, principalmente, un papel sensibilizador en pro de las NEE.

Se pretende desafiar la realidad, mediante la creación de apoyos y de competencias fundamentales requeridas para que todas las personas participantes de la labor educativa, directa o indirectamente, introduzcan en su bagaje humano, profesional y conceptual, nuevos principios de acción que les permitan ir alcanzado una educación de calidad y para la diversidad. 


\section{Antecedentes}

La diversidad se manifiesta en todo lo que se observa alrededor; sin embargo, a lo largo de la historia ha sido evidente que a los seres humanos se les dificulta aceptar las diferencias dadas entre unos y otros; el rechazo y la invisivilización de las personas con condiciones diversas es reflejo de ese comportamiento de segregación que se adopta ante ellos.

Los centros educativos no se encuentran ajenos a esta realidad, y es notorio lo complejo que resulta lograr una intervención pedagógica significativa de parte de las docentes y los docentes que atienden niños y niñas con NEE, que responda al enfoque de sus derechos y a una educación inclusiva, mediante una planificación educativa que parta de la diversidad y las necesidades de todos los estudiantes, sea cual sea su condición.

Aunque con diferencia de años, Costa Rica no ha estado alejada de la discusión planteada en el mundo en torno a la discapacidad y a la Educación Especial. Todas las propuestas surgidas por dinámicas particulares en algunos países o por discusiones en grandes organismos unilaterales como la UNESCO, y por medio de declaraciones como la de Salamanca, con la premisa "Educación para todos", han pasado a ser discutidas, analizadas y adecuadas a la realidad de Costa Rica (Dobles, 2006).

Lograr una educación para la diversidad constituye, todavía, un ideal, un imaginario en el proceso educativo. Este se ha constituido en un tópico de investigación que despierta el interés en diferentes autores y autoras, por indagar sobre las mejores alternativas y estrategias de intervención educativa, para asegurar procesos de enseñanza y de aprendizaje acordes con las particularidades de cada estudiante.

En el ámbito educativo nacional ha surgido, también, un especial interés por alcanzar una educación inclusiva de calidad, en cuya discusión se cuestionan los programas del Ministerio de Educación Pública (MEP), la descontextualización del currículo, la intervención y el papel que cumplen las familias en la educación de sus hijos e hijas, y, por supuesto, el personal docente como agente de cambio.

Para efectos de este estudio, se revisaron distintos autores que tratan esta temática, entre los que se encuentran: Avendaño et al. (2006); Cubillo, Guadamuz y Mejías (2006); Alemany (2004); Almazán (2004), cuyas investigaciones presentan, como común denominador, el mejoramiento de los procesos de enseñanza y de aprendizaje, todas se llevan a cabo desde una perspectiva o visión diferente; pero con una invitación a la reflexión y al análisis del papel desempeñado por los docentes y las docentes hasta el momento. De igual manera, coinciden en la necesidad de que sean capaces de generar cambios, que propicien una verdadera educación para la diversidad. Ante ello, las habilidades de gestión, la negociación y la intervención son esenciales para el logro de ese ideal educativo.

Al revisar las conclusiones de las indagaciones previas, se encontraron argumentos que respaldaron la importancia de la investigación, desde una visión más participativa y que involucrara a todos los sectores que intervienen en el proceso educativo, ya que, desde su implementación, se buscaba desafiar esa realidad mediante un abordaje cualitativo, profundo y reflexivo.

\section{Objetivo general}

Facilitar el proceso de formación de los docentes y las docentes que atienden niños con NEE en las escuelas de Río Ora, 26 de febrero de 1886, Lajas y el Jobo, mediante el apoyo de las estudiantes 
de Pedagogía en I y II Ciclos, Educación Preescolar y Educación Especial, para promover la gestión de recursos, la negociación y la intervención entre los actores involucrados en el proceso educativo.

\section{Objetivos específicos}

- Diagnosticar la atención de las NEE, según los actores que intervienen en el proceso educativo.

- Determinar las limitaciones en relación con la atención a las NEE que presentan docentes, estudiantes, instituciones y comunidades.

- Sistematizar la información obtenida para propiciar espacios de reflexión que generen propuestas acertadas en torno a la práctica docente, en el marco de la atención a las NEE.

- Propiciar el establecimiento del trabajo interdisciplinario en los ámbitos educativos, mediante ejercicios de gestión y de negociación realizados por los diferentes actores del proceso educativo de las escuelas de Río Ora, 26 de febrero de 1886, Lajas y el Jobo, para favorecer el proceso de inclusión de los niños y las niñas con NEE.

\section{Hacia dónde debemos mirar la diversidad como parte de la cotidianidad del aula}

Imaginemos la diversidad de un paisaje rural, montañas, ríos y biodiversidad en flora y fauna, personas con costumbres y un estilo de vida propio de la cultura guanacasteca. A buena mañana y por las tardes, niños sonrientes entre sus juegos caminando por caminos de lastre que representan historias de vida de esos pequeños pueblos, hacia el lugar en donde se aglomera toda esa diversidad: sus centros educativos.

Una diversidad tan amplia y tan compleja que parece ser imposible de integrar en la planificación educativa, alimentada por un currículo descontextualizado y emanado desde las más altas jerarquías del MEP. Pero, ¿será acaso una tarea inalcanzable?, ¿deberán, por ende, los estudiantes escolares seguir bajo un sistema de educación que fragmenta sus realidades?

Al hablar de atención a la diversidad como parte esencial dentro de la planificación educativa, afloran nuevos conceptos que brindan una orientación más clara sobre cómo lograr, precisamente, esa atención de las necesidades y los intereses del estudiantado y, por tanto, la modificación del contexto en el cual está inmerso, como son: el trabajo interdisciplinario, el enfoque multidimensional, la gestión y la negociación dentro de los procesos educativos, la intervención docente y la integración de los sectores que participan en la formación y el desarrollo de los elementos involucrados con las los estudiantes y los estudiantes: comunidad, familia y docentes.

Estos elementos son claves y evidencian que la atención a la diversidad es más que palabras, más que una propuesta, pues constituyen procesos de reflexión y de análisis desde la realidad del estudiante, y nos permiten preguntamos por qué ligar investigación con diversidad. La respuesta es muy simple: todo educador debe conocer el contexto dentro del que se encuentra inmersa la institución, pues, de lo contrario, los procesos educativos pierden significado para los niños y las niñas, quienes visualizan los contenidos como temas ajenos a su realidad, a sus vivencias. Lamentablemente, se cae en el error de continuar promoviendo un currículo descontextualizado.

Una asertiva atención a la diversidad debe iniciar, por tanto, desde la propia vivencia de la investigación del educador, de la reflexión y el análisis de su práctica docente. Motivado 
por buscar nuevas acciones de cambio que les permitan a las estudiantes y los estudiantes descubrir en los centros educativos algo más que cuatro paredes, tareas y pruebas que les resultan tediosas, llamadas de atención y rigidez por parte del maestro, además de aprendizajes memorizados y sin significado alguno. Las instituciones educativas deben representar para ellos y ellas, disfrute, interés, motivación, alegría, aprendizaje significativo y sonrisas constantes, presentes a lo largo de toda la jornada escolar y no solamente durante los recreos.

La institución debe identificarse como una promotora de unión, de trabajo en equipo, de comunicación, de diálogo y de planificación de acciones para mejorar nuestro sistema educativo, y para asegurar esa educación de calidad a la que, supuestamente, tienen derecho los niños y las niñas, la cual debe partir de una atención y de un respeto por la diversidad que caracteriza el contexto, las familias, las necesidades y los intereses del estudiantado.

Justamente, desde el principio de la atención a la diversidad, es muy importante interactuar con la persona y con el contexto inmediato en el que se desenvuelve, es reconocer e interactuar con el estudiante y su medio. Por tanto, nuestro acercamiento con esa realidad permite comprender la cultura, la familia y el entorno desde un enfoque multidimensional.

Un enfoque multidimensional tiene que ver con la forma en la que el estudiante se percibe, por lo que conceptos de autoimagen, autoestima, autocontrol, son radicales en este apartado. Las NEE vistas desde un enfoque multidimensional permiten al educador abrir un abanico de oportunidades de aprendizaje, no mirándolas como una limitación propia del estudiante, sino en función de los apoyos que el estudiante necesita de manera que el entorno deba ajustarse a ellas.

\section{Diversidad: una escuela para todos}

Cuando se habla de una escuela para todos, se hace referencia a la integración de todos los actores de los diferentes contextos con los que se relaciona el niño y la niña, como son: la comunidad, los padres de familia, las docentes y los docentes, la institución educativa y otras organizaciones gubernamentales y no gubernamentales.

En la Declaración Mundial sobre Educación para Todos, en Jomtien, Tailandia, (1990), se indica que el sistema educativo no sólo debe ser el único ente que genere los recursos para la educación, sino que debe ser necesaria “... la concertración de acciones entre el Ministerio de Educación y otros ministerios..., la cooperación entre organizaciones gubernamentales y no gubernamentales, el sector privado, las comunidades locales, los medios de comunicación, los grupos religiosos y la familia" (Guajardo, 2001, citado en Castillero, Díaz, Morales y Pino, 2002, p. 51).

En la Declaración Mundial sobre Educación para Todos, en Jomtien, Tailandia, (1990), se indica que el sistema educativo no sólo debe ser el único ente que genere los recursos para la educación, sino que debe ser necesaria “... la concentración de acciones entre el Ministerio de Educación y otros ministerios..., la cooperación entre organizaciones gubernamentales y no gubernamentales, el sector privado, las comunidades locales, los medios de comunicación, los grupos religiosos y la familia" (Guajardo, 2001. p. 149 citado en Castillero, Díaz, Morales y Pino, 2002, p. 51).

Debe rescatarse que el apoyo que cada una de estas instancias pueda brindarle a la institución está dirigido a las diferentes áreas de acción, entre otras: a la gestión de los programas educativos, financieros y administrativos. La educación es una responsabilidad 
de todos, de manera que una calidad educativa para todos los estudiantes y las estudiantes se logra mediante la satisfacción de sus necesidades, el compromiso y la responsabilidad del docente en su labor pedagógica, lo que mejoraría el desempeño en cada una de las distintas áreas en las que se desarrollan.

\section{Empoderamiento docente: concepto clave para una innovación pedagógica}

En la actualidad, aparentemente, uno de los problemas más comunes en los centros educativos costarricenses lo constituye la falta de acercamiento del cuerpo docente a la realidad en la que se desenvuelven sus estudiantes a diario; podría pensarse que este grupo se preocupan sólo por cumplir los objetivos que plantea el MEP, sin darse cuenta de los elementos que favorecen a los niños y las niñas en su aprendizaje y de los conflictos, las limitaciones y las circunstancias que les perjudican. Ante esta situación se hace necesaria una innovación pedagógica en cada uno de los centros educativos, una concienciación por parte de los maestros y las maestras sobre la importancia de conocer, indagar y visitar a las familias de los niños y las niñas, recorrer la comunidad y conocer las situaciones en que se encuentra la institución.

Con base a lo anterior, Sampa, Gómez, Codutti y Salazar (2007) comentan que el docente debe “(...) contribuir a la adquisición de saberes y conductas de participación, como agente de cambio social y promotor de actitudes de solidaridad, a través de la preparación para el desempeño de roles protagónicos y dirigenciales del sistema educativo" (J 1).; sin embargo, en la actualidad, parecieran ser pocos los educadores que reflexionan y analizan su práctica pedagógica, por tanto, no logran una transformación en las escuelas.

Por ello, este trabajo de innovación y de mejoramiento permanente en los centros educativos de Río Ora, 26 de febrero de 1886, Lajas y el Jobo, precisa adoptar una nueva manera en que el docente o la docente logre tomar en cuenta la negociación, la gestión y la intervención, los cuales son conceptos claves para lograr, con éxito, una innovación pedagógica en el centro educativo y que éstos puedan ser integrados creativamente. Pero, ¿a qué nos referimos con negociación, gestión e intervención y qué beneficios pueden obtener las docentes y los docentes con la implementación de estas concepciones?

La negociación es entendida “(...) como el proceso dinámico en el cual dos o más actores, en conflicto posible o manifiesto, o con intereses divergentes, entablan una comunicación para generar una solución aceptable de sus diferencias que se explicita en un compromiso" (IIPE/UNESCO, 2000, p. 16), por tanto, se explica como la acción en la que dos personas se reúnen para dialogar, intercambiar, plantear sus intereses y presentar sus argumentos, con la intención de llegar y decidir un acuerdo que diseñe un objetivo compartido.

En cuanto al segundo concepto antes mencionado "gestión", según el Instituto Internacional de Planeamiento de la Educación (2000), “... la gestión educativa es vista como un conjunto de procesos teórico-prácticos integrados horizontal y verticalmente dentro del sistema educativo, para cumplir los mandatos sociales" (p. 3), esa capacidad es la que todo docente debe llevar a cabo cuando está bajo la dirección de un grupo o una institución, con lo que se lograría la superación y el bienestar de ese ente.

De igual forma, el concepto gestión se liga con la idea del fortalecimiento, la integración y la realimentación del sistema, lo que propicia al educador una multiplicidad de saberes pedagógicos, principios útiles para la acción, juicios de valor y colaboración de diversas personas. Esta colaboración se convierte en el enunciado de una cultura escolar orientada a ofrecer a la institución una visión compartida, acerca de hacia dónde se quiere ir y cuáles son los proyectos y los principios educativos que 
se quieren promover, con los que se facilite la comprensión, la planificación, la acción y la reflexión de lo que se quiere lograr (Instituto Internacional de Planeamiento de la Educación, 2000).

El tercer y último concepto "intervención” “... es el elemento nuclear de todo proceso de enseñanza-aprendizaje. Cualquier intervención parte de una evaluación previa, a la que sigue un entrenamiento estratégico que finaliza con una evaluación final, útil para contrastar la eficacia de todo el procedimiento desarrollado" (Grupo de Investigación ADIR. Universidad de Oviedo, s. f., I 1). Es fundamental que en todo proceso de enseñanza y de aprendizaje el docente o la docente ejecute, adecuadamente, su intervención con sus estudiantes, para que se obtenga un aprendizaje interesante, duradero y significativo, y lograr, así, en ellos, una respuesta y una motivación asertiva, mediante la propuesta de metas y de objetivos para un futuro.

Se puede decir, de acuerdo con Sañudo (2001), que la intervención educativa implica detectar una problemática o una situación de la realidad educativa que puede ser cambiada, en la que la mediación del docente, desde un punto de vista específico y estructural, le permite encontrar una solución al fenómeno o evento educativo.

\section{La interdisciplinariedad desde la formación docente}

La atención de la diversidad no es una tarea que resulte sencilla para los docentes y las docentes, a causa de que implica una planificación asertiva que debe tomar en cuenta las características del contexto en el que está inmerso el centro educativo, así como las particularidades de cada estudiante, sus necesidades e intereses. De ahí la importancia del diagnóstico educativo; no obstante, no basta con conocer esa compleja realidad, sino que se debe llevar a la práctica, a la cotidianidad del aula.

El cuestionamiento que se presenta, por tanto, es cómo concretar las acciones que le permitan al cuerpo docente ejecutar una intervención para la diversidad, en la que el estudiantado reciba una educación de calidad; en igualdad de condiciones; que respete su realidad, y que la integre en la planificación como estrategia que fortalezca los procesos educativos. Es importante el trabajo interdisciplinario, el trabajo en equipo del personal docente y el aporte que cada disciplina pueda brindar para lograr, precisamente, una planificación que atienda los principios de la diversidad.

En la dinámica del aula escolar se vivencia, día a día, el aumento de la población estudiantil, lo que trae consigo la diversidad de características tanto físicas como individuales, lo cual permite ser único dentro del grupo con el que se interactúa. Esto da paso a que los docentes y las docentes tomen en cuenta la diversidad como un reto en su currículo. Por lo anterior, Devalle de Rendo y Vega (1999) comentan que en el ejercicio profesional docente, se deben tener en cuenta ciertos criterios importantes, tales como: el respeto por la diversidad, el fomento y la coordinación de las actividades en grupo, la elaboración de los materiales didácticos específicos, la disposición, la incorporación y la capacitación de las familias para realizar un trabajo en conjunto, en una adecuada atención a la diversidad.

Del mismo modo, el ejercicio profesional docente debe comprender la unión de los diferentes actores de la sociedad, que signifique un acto de formación e información de manera interactiva, para poder responder, de una óptima manera, a la diversidad presente en el aula (Devalle de Rendo y Vega, 1999). Es necesario que las docentes y los docentes tengan una formación interdisciplinaria, y que se fortalezcan con los múltiples conocimientos de cada disciplina específica para poder, de esta forma, solventar el problema de una formación determinada y no extendida a otras ramas y otros conocimientos. 


\section{Trabajo interdisciplinario: una herramienta para la atención a la diversidad}

Es evidente que cada disciplina tiene su ideología y su base para orientar los procesos educativos de acuerdo con los distintos niveles pedagógicos por los que, año con año, pasan las estudiantes y los estudiantes; pero la realidad no puede ser fragmentada porque, de lo contrario, los procesos educativos pierden significado y se vuelven una realidad abstracta para el estudiantado.

La formación curricular por disciplinas, en la realidad escolar, ocasiona que las los docentes y los docentes fragmenten el desarrollo integral de sus estudiantes, lo que irrespeta a los niños y las niñas, quienes pasan de un nivel al otro sin la adecuada articulación. Asimismo, cuando no existe trabajo en equipo o interdisciplinario, el grupo de docentes, se ve forzado a enfrentarse a los retos escolares de manera individualizada, lo que obstaculiza una adecuada atención integral del estudiante.

El nuevo paradigma de atención a la diversidad implica una nueva organización de los centros educativos, la adquisición de habilidades para gestionar y para negociar con diferentes profesionales y demás actores del currículo, los que necesitan apoyo para alcanzar, con éxito, esa planificación integral y la puesta en práctica de las acciones pedagógicas necesarias para brindar una educación que fomente las capacidades individuales y atienda las necesidades estudiantiles.

En relación con lo anterior, Campos, García y Rojas (2002) expresan que “...actualmente, se considera el trabajo en equipo en las escuelas como uno de los elementos para impulsar el mejoramiento de la calidad de la enseñanza y el desarrollo profesional de los educadores" (p. 295). Mediante el trabajo interdisciplinario no sólo se busca lograr una planificación que atienda la diversidad de contexto estudiantil y familiar en el que está inmersa la institución, sino que, además, permita a las docentes y los docentes enriquecer sus conocimientos en relación con otros disciplinas.

En este sentido, durante la investigación realizada, se llevaron a cabo observaciones y trabajos en las aulas, desde su disciplina. Esos aportes enriquecieron la visión de cada una de las educadoras y los educadores, así como sus conocimientos, lo cual pudo contribuir a mejorar, en gran medida, la calidad en la educación y la forma de intervención docente, pues permitió lograr conciencia de que cada uno tiene sus habilidades y sus destrezas educativas; pero, también, sus debilidades. Estas últimas pudieron reforzarse mediante el trabajo interdisciplinario, cooperativo y en equipo.

Puede afirmarse que no es simplemente una hipótesis, sino un hecho vivido desde la experiencia de la investigación, pues, en la realidad educativa, las estudiantes de Preescolar, Educación Especial y I y II Ciclos se encontraron observando las dinámicas del aula, cada una haciendo aportes importantes desde su perspectiva, y lo más importante, enriqueciendo sus experiencias y sus conocimientos.

\section{Reconocimiento de la diversidad en la relación investigadoras-sujeto de estudio e interacción con la realidad}

Ya hemos logrado analizar algunos puntos importantes desarrollados en la investigación. Ahora es esencial reflexionar acerca de cómo se manifestó esa relación entre las investigadoras y el sujeto de estudio, para, precisamente, poder comprender la diversidad que caracteriza a las poblaciones del estudio y que, sin duda, deben tomarse en cuenta dentro de la planificación educativa. 
Siguiendo los principios de la investigación-acción, el involucrar de manera activa al sujeto de estudio con todos los actores del proceso fue muy relevante, ya que esto permitió mantener cambios más a mediano y a largo plazo. Es decir, las investigadoras no llegaron al contexto a planificar una propuesta previamente diseñada para luego marcharse, pues eso no tendría sentido, ya que ello no podría generar cambios significativos; por el contrario, una de las premisas fundamentales para la investigación-acción fue generar acciones de cambio y lograr un impacto en el contexto de estudio.

Se pudo llegar a ese cambio estimulando e incentivando una participación activa de todos los involucrados y realizando visitas a los hogares, entrevistas, sesiones de trabajo, entre otras. Lo anterior facilitó el mantener una comunicación más flexible, en confianza, recabando datos reales y significativos de las costumbres, estilos de vida, problemáticas, sentimientos y preocupaciones de las personas, para lograr, así, conocer, comprender y contextualizar, asertivamente, esa diversidad que les identifica. De alguna manera, las investigadoras llegaron a un punto en el que formaron parte de ellos y de su realidad.

Ahora bien, hay que preguntarse quién fue ese sujeto de estudio que brindó los datos necesarios para reconocer la diversidad. Posiblemente, durante el desarrollo de la lectura se hizo evidente cuál fue el principal actor: los docentes, esto porque en ellos recayó la mayor parte de la responsabilidad por asegurar una adecuada atención a la diversidad y, por ende, una mayor calidad en los procesos formativos.

Sin embargo, otros sectores cumplieron un papel esencial, y más si se habla de interdisciplinariedad, trabajo coordinado y en equipo. Éstos fueron: la familia y la comunidad, a causa de la gran influencia que ejercen sobre la formación de los niños y las niñas, quienes fueron considerados, también, como parte de la investigación, pues se constituyeron, prácticamente, en los principales protagonistas del proceso, ya que son quienes reflejan la diversidad del contexto en el que se desenvuelven.

Estos sectores, en la investigación, tienen identidad. Dejarlos en el anonimato constituiría un irrespeto al aporte valioso que dieron al estudio; además, sin ellos el proceso no se habría logrado y el enriquecimiento de la experiencia en los niveles profesional y personal para las investigadoras, sin duda, no sería el mismo. A continuación, se detalla el nombre de las comunidades, su ubicación geográfica y el circuito de los centros educativos (véase Tabla 1).

Tabla 1

Ubicación de los centros educativos

\begin{tabular}{llccc}
\hline \multirow{2}{*}{$\begin{array}{c}\text { Nombre de la } \\
\text { comunidad }\end{array}$} & \multicolumn{2}{c}{ Ubicación geográfica } & Nombre de la escuela & $\begin{array}{c}\text { Dirección Regional de } \\
\text { Nicoya }\end{array}$ \\
\cline { 2 - 5 } & Cantón & Distrito & & Circuito \\
\cline { 3 - 5 } Rio Ora & Nandayure & Zapotal & Centro Educativo Río Ora & 07 \\
Matambú & Hojancha & Primero & Centro Educativo 26 de febrero de 1886 & 05 \\
Lajas & Hojancha & & Centro Educativo Lajas & 05 \\
El Jobo Norte & Nicoya & Nicoya & Centro Educativo El Jobo & 01 \\
\hline
\end{tabular}

Nota. Elaborada por Delgado, Hernández, León, Morera C., Morera V. y Obregón, 2008. 
La diversidad con la que las investigadoras se encontraron en los mencionados contextos fue amplia y variada, esto llevó a orientar, poco a poco, la investigación al punto clave que era, precisamente, la atención a la diversidad. En un inicio, el estudio estaba orientado a las necesidades educativas especiales, no obstante, a causa de esa relación entre las investigadoras y los sujetos participantes en el estudio, al final del proceso investigativo, se concibió más como una relación sujetos investigadores-sujetos investigados. Esto por los aportes valiosos y la participación que se logró por parte de los últimos, lo cual permitió comprender la necesidad de una orientación del problema de investigación hacia lo que los participantes apuntaban, según sus necesidades.

La investigación-acción permitió la construcción de nuevos conocimientos para las investigadoras y los grupos involucrados, la movilización, el reforzamiento y un mejor empleo de los recursos disponibles, con respecto al análisis crítico de las necesidades y las opciones de cambio. Es por ello que la interacción con los participantes y las participantes en el campo de estudio accedió al intercambio de información relevante, lo que sirvió como sustento para generar una propuesta de cambio en la que se planificaron acciones viables.

La planificación de esas acciones viables se logró mediante esa contextualización real de cada una de las comunidades, conociendo a las familias, manteniendo acercamientos significativos con los estudiantes, planificando reuniones comunales, visitando los hogares, compartiendo con ellos sus costumbres y tradiciones, conociéndolos realmente, manteniendo diálogos y convivios con los docentes y las docentes.

Se llevó a cabo una variedad de experiencias que afectó no sólo a los participantes y las participantes, sino, también, a las investigadoras, quienes lograron desarrollar aún más una visión integral y completa de lo que implica reconocer la diversidad dentro del campo educativo.

La investigación-acción, permitió no sólo transformar el contexto y a los participantes, sino que tomó en cuenta el papel significativo de las investigadoras, y mediante esa participación activa fue que ellas también se transformaron, ampliaron conocimientos, comprendieron asertivamente el problema de estudio, porque lo vivieron junto con los sectores involucrados, con lo que lograron desarrollar experiencias de aprendizaje y de formación verdaderamente significativas.

La experiencia de la investigación tuvo resultados muy positivos para todos los sectores participantes, pues movió, en cada uno de ellos, diversos sentimientos y acciones con miras a mejorar la calidad en la educación, así como a asegurar una adecuada atención a la diversidad. Por tanto, se presenta un resumen del análisis e interpretación de los datos obtenidos en la investigación, para efectos de claridad, dicho análisis se trabajó en tres fases: diagnóstica, propuesta y ejecución.

\section{Diagnóstica: indagando, reflexionando y analizando la realidad}

Mediante los instrumentos aplicados a los participantes en la fase diagnóstica de esta investigación, se pretendió un acercamiento, en primera instancia, al contexto y a la situación en relación con la atención a las $\mathrm{NEE}$, para responder a los objetivos específicos antes mencionados.

Esos instrumentos, utilizados en el diagnóstico y aplicados en los diferentes sectores, arrojaron diversos datos acerca de la situación general del proceso educativo de las instituciones de Río Ora, 26 de febrero de 1886, Lajas y El Jobo. 
La identificación de las situaciones presentes en cada sector puede apreciarse en la Figura 1.

\section{LO QUE ENCONTRAMOS}

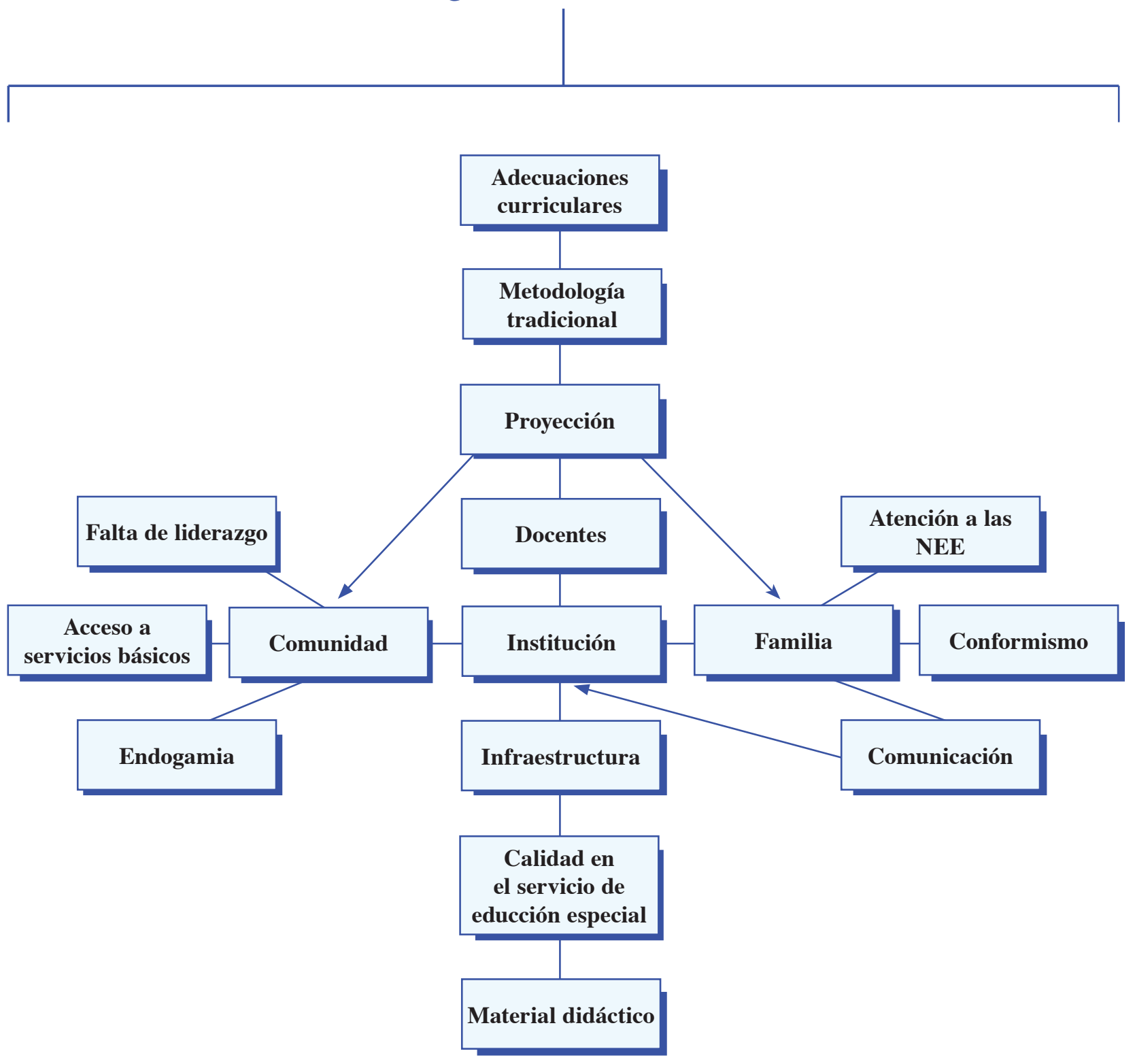

Figura 1. Características de cada sector.

Nota. Elaborada por Delgado et al., 2008. 


\section{Propuesta: buscando soluciones}

Esta fase consistió, principalmente, en la presentación del diagnóstico al personal docente. Como característica esencial dentro de la investigación-acción, mediante la devolución de datos de la primera fase del estudio se pretendía informar a los maestros de las fortalezas y las necesidades identificadas, e incentivar, al mismo tiempo, la búsqueda de posibles líneas de acción.

Esta fase implicó una explicación detallada, ya que los docentes mantenían una concepción de que el trabajo de la propuesta sería efectuado por las estudiantes de la UNA; por tanto, fue indispensable aclarar que uno de los objetivos de la investigación era propiciar en ellos las habilidades de gestión, de negociación y de intervención en la solución de la problemática que se presenta en el quehacer educativo.

La Figura 2 representa los pasos que se dieron en esta fase.

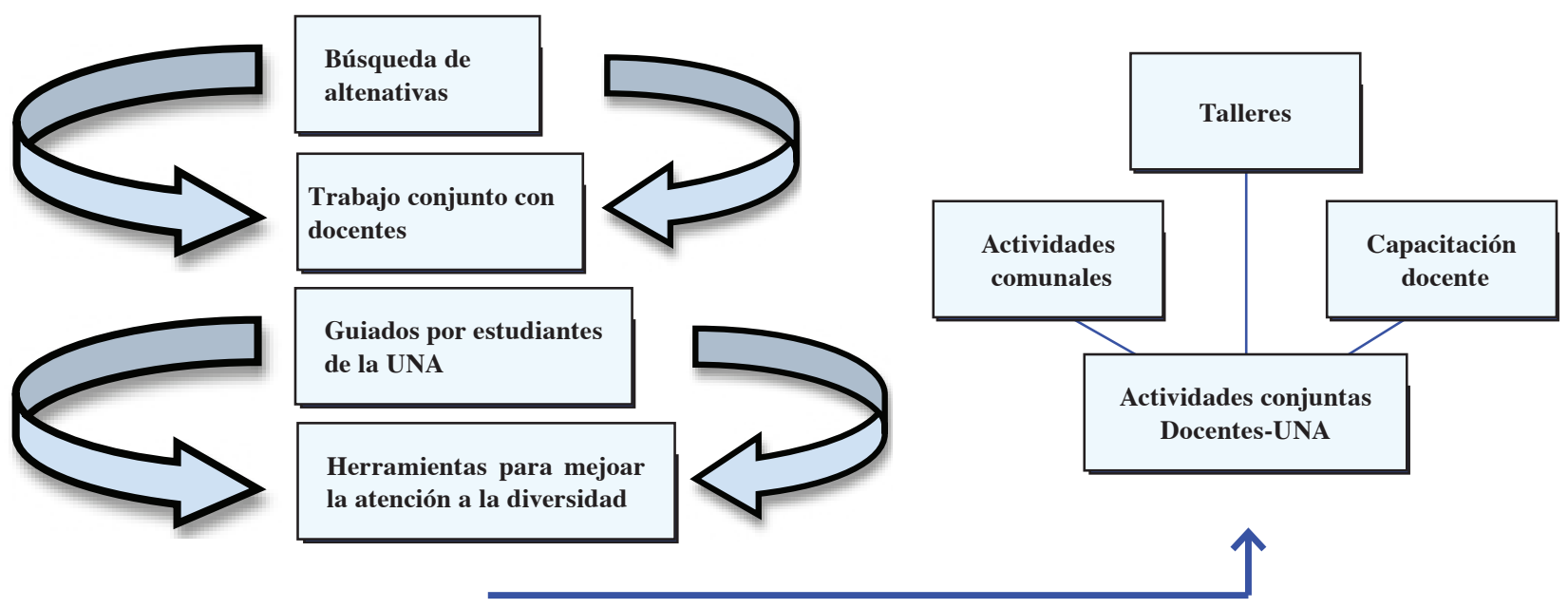

Figura 2. Propuesta.

Nota. Elaborada por Delgado et al., 2008.

\section{Ejecución: manos a la obra}

Una vez planificadas y coordinadas las acciones, se presentó la ejecución de las actividades, tomando en consideración que no en todos los centros educativos se logró una verdadera gestión y negociación con las docentes y los docentes, ya que se evidenció poco interés por parte de éstos. Con base en lo anterior, se consideró de vital importancia involucrar, activamente, a los demás sectores participantes del seminario: comunidad, familia, docentes y estudiantes, ya que uno de los fines esenciales dentro de la investigación-acción es impactar en la realidad de estudio y lograr, a la vez, que los docentes visualicen acciones más concretas en la búsqueda de la integración de los contextos, con el objetivo de mejorar la atención a la diversidad y, por ende, la calidad educativa.

El proceso de ejecución se realizó de acuerdo con los diferentes entornos y actores; se enfrentó una serie de obstáculos para la realización de acciones. Por ejemplo, en algunos casos, la accesibilidad a las comunidades durante la época lluviosa provocó la suspensión de algunas actividades programadas. Además, los sentimientos de rechazo por parte de docentes provocó que se suspendieran, en varios casos, las actividades, o bien, que no participaran por diferentes 
circunstancias. La poca presencia e inadecuada supervisión, por parte del MEP, constituyó otro contratiempo.

Sin embargo, se dieron procesos que facilitaron en diferentes acciones el fortalecimiento y el cumplimiento de los objetivos del proyecto, entre ellos: el modelamiento de métodos de trabajo, las intervenciones pedagógicas en las aulas, las reuniones con líderes comunales, los procesos de gestión y negociación con municipalidades e instituciones estatales y privadas, los bingos, los talleres para padres y madres, docentes y estudiantes.

Un aspecto importante por tomar en cuenta dentro del accionar fue la modificación realizada al enfoque del proyecto, ya que en un inicio era la atención a las necesidades educativas especiales, la cual fue realizada a causa del abanico de situaciones diversas, lo que provocó un enfoque más amplio y apegado a la realidad, como es la atención a la diversidad.

Para la realización de las acciones en la fase de ejecución se tomaron en cuenta dos tipos de recursos, a saber:

- Internos. La experiencia docente, la apertura por parte de la comunidad, la experiencia y la organización de los comités comunales y la motivación y disponibilidad de las familias y estudiantes.

- $\quad$ Externos. Las instituciones de Gobierno, las municipalidades, la UNA con el respaldo del personal, el acceso a las profesionales y los profesionales de las diferentes disciplinas, entre otros.

El análisis regional consta de la sistematización de datos obtenidos, de las características encontradas por sectores comunes en las cuatro escuelas participantes del proyecto, con el objetivo de realizar una caracterización real mediante una pequeña muestra de la Región Educativa de Nicoya de los circuitos 01,05 y 07 . Con un total de 172 escuelas distribuidas en 8 circuitos, de las cuales un $60 \%$ son escuelas unidocentes.

Este análisis resulta esencial dentro de la sistematización de los informes, lo que facilita generar un espacio de reflexión sobre la calidad educativa que, aparentemente, tiene el país, el cual se enorgullece por los grandes logros alcanzados como la educación gratuita y obligatoria, la disminución de los niveles de deserción y el apoyo al estudiantado de escasos recursos. No se debe negar que el país ofrece mejores condiciones educativas en comparación con otros países centroamericanos, lo que permite tener una población con un mayor nivel académico; sin embargo, la investigación hace relucir lo que pareciera una falta de interés de las autoridades del Gobierno y del MEP hacia aquellas poblaciones rurales que enfrentan situaciones adversas, y que han quedado en el olvido a los ojos de las direcciones regionales educativas.

\section{Principales conclusiones}

Después de haber realizado la investigación, se pueden rescatar, como principales, las siguientes conclusiones:

Se determinó, en las familias, la existencia de una concepción de educación pasiva, es decir "aprender lo necesario para sobrevivir", lo cual es una ideología que surge a partir de la historia y la cultura de la comunidad, y que se refleja en una pobre visión a futuro, ya que lo que sus padres vivieron es lo que esperan de sus hijos. Además, es notable la falta de involucramiento en el proceso de aprendizaje de los niños y las niñas, a causa de las condiciones adversas de la vida, pues no 
cuentan con el tiempo, las habilidades ni los recursos económicos, y le dejan la responsabilidad de la educación de sus hijos e hijas al docente.

El encontrarse con un grupo de profesionales interesados por sus inquietudes, necesidades e intereses, hizo que las diferentes comunidades y familias mostraran bastante colaboración y apertura durante el desarrollo del proyecto, que mantuvieran una relación bilateral y que brindaran muchos conocimientos acerca de estos sectores.

Debe realizarse una formación universitaria de tipo interdisciplinaria, que permita a las estudiantes y los estudiantes aprender sobre las metodologías, ritmos de trabajo e ideas sobre las demás disciplinas. Sin embargo, representa un reto, ya que desde la formación escolar el estudiante aprende a trabajar por y para sí mismo. El docente, a su vez, debe contar con una formación en valores que le permita dar una adecuada atención a la diversidad, y logre trabajar con diferentes etnias, religiones, inteligencias y contextos.

El sistema educativo nacional no debe centrarse únicamente en los centros educativos urbanos, pues existen muchas poblaciones no convencionales con necesidades muy distintas, pues el MEP se centra en los sectores urbanos, y las docentes y los docentes rurales asumen la propuesta curricular intacta, sin realizar modificaciones o adaptaciones que tomen en cuenta las características, las necesidades y las limitaciones de estas zonas, lo que genera problemas de indisciplina, desmotivación e inadecuada atención a la diversidad.

Finalmente, los niños y las niñas mostraron interés en suplir sus necesidades nutricionales y emocionales, aspectos no tomados en cuenta dentro de la planificación docente por la descontextualización del currículo, la que no cuenta con una adecuada atención a la diversidad. No obstante, estos manifiestan aspiraciones profesionales, las cuales, muchas veces, se ven limitadas por la visión de sus padres y las condiciones económicas y geográficas de la región. Es evidente que los estudiantes son un reflejo de cada uno de los contextos en que se desenvuelven, lo que viene a generar un círculo vicioso, que se repite de generación en generación.

\section{Reflexiones finales}

Es necesario crear redes de apoyo directas con las universidades y demás instancias encargadas de formar educadores, de manera que estas promuevan, mediante los diferentes trabajos de campo e investigación, un apoyo para el MEP en la búsqueda de alternativas para mejorar la educación costarricense.

El docente debe rescatar el protagonismo que merece como gestor de cambio desde lo local, de modo que este sea capaz de negociar y gestionar las posibles alternativas que le permitan dar una correcta atención a la diversidad dentro de su contexto educativo.

El concebir la educación desde una disciplina única es imposible, especialmente si se quiere ver desde un enfoque integral, por tanto, si se considera que las docentes y los docentes son formados en distintas disciplinas, es necesario que el MEP vele porque cada institución educativa cuente con los servicios de apoyo necesarios para atender a la diversidad existente en cada población estudiantil.

Es importante propiciar estrategias metodológicas, apoyo, guías, capacitaciones y demás actividades que brinden confianza a las docentes y los docentes, de manera que se sientan respaldados, seguros en su desarrollo profesional y laboral. Asimismo se requiere crear actividades que generen capacidades de gestión en la zona, de modo que cada comunidad logre empoderarse en la búsqueda de alternativas de solución para las sus diferentes necesidades. 
Se debe formar docentes menos técnicos y más profesionales, y motivar a aquellos que están en constante actualización, que aprenden conforme avanzan sus años laborales y que se sienten comprometidos con su deber. Es necesaria una evaluación del modelo pedagógico en que se están formando los futuros docentes, de manera que el que se adopte motive al estudiante a crear una propuesta educativa que genere un cambio en la sociedad.

Hay que lograr la autorreflexión en el docente desde sus años de formación, y sembrar la idea del maestro como actor de cambio, el cual debe estar en constante autoanálisis para mejorar su práctica laboral a partir de sus logros y errores.

Es necesario generar algunas actividades, como por ejemplo el turismo rural, que permitan explotar, en armonía con la naturaleza y la comunidad, la gran riqueza en flora y fauna de las zonas, y lograr, así, generar ingresos mediante actividades recreativas que contemplen las costumbres y las tradiciones de los pueblos.

El trabajo de los docentes y las docentes debe ser interdisciplinario y en equipo, de manera que se no se enfrenten ante retos escolares de manera individualizada, y obstaculicen la adecuada atención integral del estudiante.

Para hablar de atención a la diversidad desde la interdisciplinariedad se debe, entonces, hablar de trabajo en equipo; se debe pensar en un currículum más flexible que permita a los docentes y las docentes, dentro de su formación universitaria, compartir experiencias entre las diferentes carreras y enriquecer su formación integral, mediante la relación directa con el estudiantado, la familia, la escuela y la comunidad.

Es responsabilidad de las universidades, mediante la extensión, la docencia y la investigación, formar profesionales de cambio, reflexivos, con habilidades de gestión en comunidades, de trabajo cooperativo y amantes de la investigación desde su quehacer profesional.

\section{Referencias bibliográficas}

Alemany, I. (2004). Las actitudes del profesorado ante el reto de integrar a alumnos con N.E.E. Una propuesta de trabajo. Revista Polibea, N 72, Recuperado 03 Mayo, 2008 de http://pasoapaso. com.ve/CMS/index.php?option=com_content\&task=view\&id=581\&Itemid=421\&limit=1\&li mitstart $=0$

Almazán, L. (2004). Los cambios actitudinales hacia la integración escolar desde una perspectiva de los alumnos. Revista Fuentes, 5. Recuperado el 22 Julio, 2008 de http://institucional.us.es/ revistas/revistas/fuente/pdf/numeros/5/07\%201os\%20cambios.pdf

Avendaño, P., Brenes, A., Cubillo, R., Dobles, C., Navarrete, L., Navarro, T., Solano, S. \& Yglesias, A. (2006). Funciones del docente de apoyo en los servicios de Educación Especial. San José, Costa Rica: Fundación mundo de oportunidades. Centro Nacional de Recursos para la Inclusión Educativa (CENAREC). Departamento de Investigación.

Campos, N., García, N. y Rojas, M. (2002). La administración escolar. San José, Costa Rica: Editorial Universidad de Costa Rica. 
Castillero, A., Díaz, J., Morales, F. \& Pino, I. (2002). Gestión y supervisión en el centro de educación básica. Cartago, Costa Rica: Editorial Coordinación Educativa y Cultural Centroamericana (CECC).

Cubillo, L., Guadamuz, P. \& Mejías, A. (2006). Los procesos de integración de personas con necesidades educativas especiales en el sistema regular, en la Dirección Regional de Heredia, circuito educativo 01. Aproximación Evaluativa. Heredia, Costa Rica: Universidad Nacional. Documento no publicado.

Delgado, P., Hernández, G., Morera, L., Morera, S \& Obregón, R. (2009). Negociación, gestión e intervención de las estudiantes de las carreras de Educación Especial y Educación Preescolar de la Sede Central y la Sede Chorotega de la Universidad Nacional, en los procesos formativos de los docentes de las escuelas Río Ora, 26 de febrero de 1886, Lajas y el Jobo, del circuito 01, 05 y 07 de la provincia de Guanacaste que atiende niños con necesidades educativas especiales. (Trabajo Final de Graduación inédito de Licenciatura en Enseñanza Especial), Universidad Nacional (División de Educación Básica, CIDE), Heredia, Costa Rica.

Devalle de Rendo, A. \& Vega, V. (1999). Una escuela en y para la diversidad. São Paulo, Brasil: Aique Grupo Editor.

Dobles, C. (2006). Negociación, Gestión e Intervención interdisciplinaria en los procesos formativos de los docentes de las tres carreras de la División de Educación Básica y las sedes regionales de la UNA. Proyecto de extensión FIDA 06-05. Heredia, Costa Rica: Universidad Nacional.

Grupo de investigación ADIR, Universidad de Oviedo (s. f.). Intervención educativa. Recuperado el 17 de marzo de 2009 de http://www.grupocerpa.com/int_edu.php

IIPE/UNESCO. (2000). Una cultura de consensos. En P. Pozner (Ed.). Negociación. Módulo 6: diez módulos destinados a los responsables de los procesos de transformación educativa, (pp. 15-18). Buenos Aires: Autor. Recuperado el 10 Octubre, 2008 de http://www.usebeq. sep.gob.mx/Pilar_Pozner/modulo06.pdf

Instituto Internacional de Planeamiento de la Educación. (2000). Gestión educativa estratégica. Recuperado 10 Agosto, 2008 de http://www.gestionescolar.cl/userfiles/ p0001\%5cfile\%5cficha 56 gestion\%20educativa\%20estrat\%c3\%a9gica.pdf

Sañudo, L. (2001). El papel de la intervención educativa en la transformación de la práctica docente. Recuperado el 6 de setiembre del 2008 de http://educar.jalisco.gob.mx/01/01Entrev.html

Zampa, D., Gómez, A., Codutti, T. y Zalazar, I. (2007). La negociación y la mediación en la formación docente. Recuperado el 03 Marzo, 2008 de http://www.nuestraldea.com/ negociacion.html 